\title{
A Characterization and Recurrence Relations of Moments of the Size-Biased Power Function Distribution by Lower Record Values
}

\author{
Shakila Bashir ${ }^{1} \&$ Mujahid Rasul ${ }^{2}$ \\ ${ }^{1}$ Department of Statistics, Forman Christian College a Chartered University, Lahore, Pakistan \\ ${ }^{2}$ Department of Statistics, Forman Christian College a Chartered University, Lahore, Pakistan \\ Correspondence: Shakila Bashir, Department of Statistics Forman Christian College a Chartered University, Lahore, \\ Pakistan. E-mail: shakilabashir@fccollege.edu.pk
}

Received: April 25, 2018 Accepted: May 10, 2018 Online Published: June 26, 2018

doi:10.5539/ijsp.v7n5p1 URL: https://doi.org/10.5539/ijsp.v7n5p1

\begin{abstract}
A variety of research papers have been published on record values from various continuous distributions. This paper investigated lower record values from the size-biased power function distribution (LR-SPFD). Some basic properties including moments, skewness, kurtosis, Shannon entropy, cumulative distribution function, survival function and hazard function of the lower record values from SPFD have been discussed. The joint probability density function (pdf) of $n^{\text {th }}$ and $m^{\text {th }}$ lower record values from SPFD is developed. Recurrence relations of the single and product moments of the LR-SPFD have been derived. A characterization of the lower record values from SPFD is also developed.
\end{abstract}

Keywords: SPFD, PFD, pdf' cdf, LR-SPFD, record values

\section{Introduction}

In the environmental and ecological work, observations usually fall in the non-experimental, non-replicated, and nonrandom categories. The problems like model specification and interpretation of data has special importance and great concern. The theory of weighted distributions provides a unique approach for these problems. Weighted distributions are applied in theory and applications of observational studies where biased data arise. Specifically, weighted distributions have applications in study of wildlife and fish populations that should motivate of those researchers who have an interest in environmental issues. Size-biased is one of the most commonly used type of various weight functions.

In this article we are introducing the lower record values from size-biased power function distribution (SPFD). Power function is a well known continuous distribution and a lot of work has been done on the record values from various continuous distributions. But in this article we weight the power function distribution named as size-biased power function distribution and derived record value from size-biased power function distribution. Some basic properties, recurrence relations for single and product moments, a characterization of the lower record values from SPFD are discussed.

Any observation is said to be a record value if its value is greater than or less than all the preceding observations. Chandler (1952) introduced the record value theory. Ahsanullah (1984) discussed the linear prediction of record values for the two parameter exponential distribution. Afterward various work on record values have been done including Ahsanullah (1986) rectangular distribution, Ahsanullah and Houchens (1989) Pareto distribution. Ahsanullah (1992) record values of independent and identically distributed continuous random variables, Balakrishnan and Chan (1993) Rayleigh and Weibull distribution, Balakrishnan et al. (1995) logistic record values, Ahsanullah and Bhoj (1996) Type-I extreme value distribution, Balakrishnan and Chan (1998) normal records, Sultan et al. (2008) gamma distribution based on record values, Teimouri and Gupta (2012) Weibull record values, Sultan (2010) inverse Weibull lifetime model, Shakil and Ahsanullah (2011) ratio of two independently distributed Rayleigh random variables, Bashir and Ahmad (2014) inverse Gaussian distribution, Bashir, Ahmed and Ahmad (2014) two sided power distribution.

Raqab (2000) established the recurrence relations for the moment of record values. Khan and Zia (2009) gave some recurrence relations satisfied by single and product moments of upper record values from Gompertz distribution and also presented a characterization. Ahsanullah (2010) derived the $r^{\text {th }}$ concomitants and joint distribution of $r^{\text {th }}$ and $s^{\text {th }}$ concomitants of record values from the bivariate pseudo-Weibull distribution. He derived recurrence relation for the single moments.

Although lots of research had been done on all well known various continuous distributions but the work on record values from size-biased distributions started by the paper, Bashir and Ahmad (2014) developed record values from the size biased Pareto distributions and derived its various properties including a characterization. Bashir and Akhtar (2014) developed record values from size-biased student's t distribution. Bashir and Rasul (2015) developed record values from 
the weighted rectangular distribution. Bashir and Ahmad (2015) developed recurrence relations for single and product moments of record values from size-biased Pareto distribution. The pdf of the $n^{\text {th }}$ Lower record values is

$$
f_{n}(x)=\frac{[H(x)]^{n-1}}{\Gamma(n)} f(x), \quad-\infty<x<\infty
$$

where, $H(x)=-\ln F(x), \quad 0<F(x)<1$.

The joint pdf of $n^{\text {th }}$ and $m^{\text {th }}$ lower record values is

$$
\begin{gathered}
f_{n, m}(x, y)=\frac{[H(x)]^{m-1} h(x)[H(y)-H(x)]^{n-m-1}}{\Gamma(m) \Gamma(n-m)} f(y), \quad-\infty<x<\infty \\
h(x)=\frac{f(x)}{F(x)}
\end{gathered}
$$

The conditional pdf of lower record values

$$
f\left(X_{L(n)}=y_{n} / X_{L(m)}=x_{m}\right)=\frac{[H(y)-H(x)]^{n-m-1}}{\Gamma(n-m)} \frac{f(y)}{F(x)}, \quad-\infty<y<x<\infty
$$

For $n=m+1$, in (3)

$$
f\left(y_{m+1} / X_{L(m)}=x_{m}\right)=\frac{f\left(y_{m+1}\right)}{F\left(x_{m}\right)}, \quad-\infty<y<x<\infty
$$

Ahsanullah and Lutful-Kabir (1974) developed a characterization from power function distribution. Saran and Pandey (2004) developed recurrence relations for moments, characterization and estimated parameters of a power function distribution by $k^{\text {th }}$ record values. Lim and Lee (2013) provided a characterization of the power function distribution by lower record values. Ahsanullah, et al (2013) provided a new characterization of power function distribution based on lower record values. The probability density function (pdf) of the power function distribution (PFD) is

$$
f(x)=\frac{\beta x^{\beta-1}}{\alpha^{\beta}}, \quad 0<x<\alpha
$$

Where $\beta>0$ shape parameter and $\alpha>0$ scale parameter. The $m^{\text {th }}$ moments of the power function distribution is

$$
\mu_{m}^{\prime}=\frac{\beta \alpha^{m}}{(m+\beta)}
$$

The size-biased power function distribution (SPFD) is

$$
f(x)=\frac{(\beta+1) x^{\beta}}{\alpha^{\beta+1}}, \quad 0<x<\alpha
$$

The cumulative distribution function (cdf) of SPFD is

$$
F(x)=\frac{x^{\beta+1}}{\alpha^{\beta+1}}
$$

\section{Record Values from the Size-Biased Power Function Distribution}

Pdf of the lower record values from size-biased power function distribution (LR-SPFD) by using equation (7) and (8) in equation (1),

$$
f_{n}(x)=\frac{(\beta+1)^{n} x^{\beta}[-\ln (x / \alpha)]^{n-1}}{\alpha^{\beta+1} \Gamma(n)}, \quad 0<x<\alpha
$$

Where $\beta>0$ shape parameter and $\alpha>0$ scale parameter 


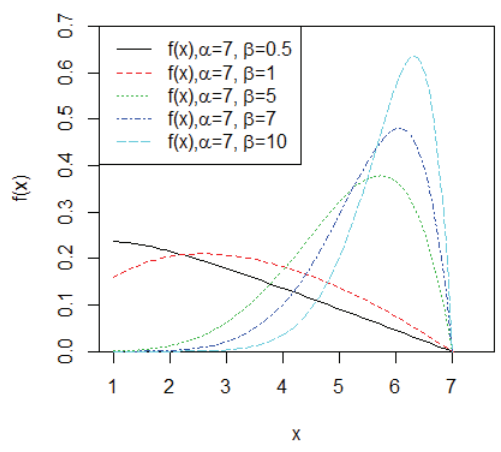

Figure 1. pdf graph for $n=2$

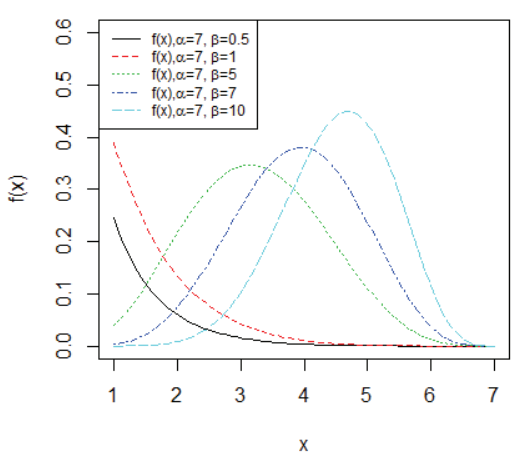

Figure 2. pdf graph for $n=5$

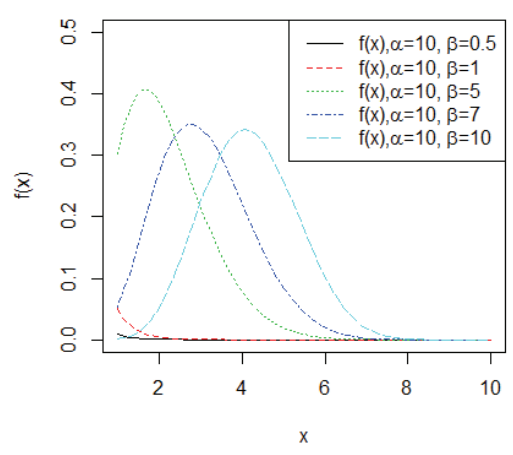

Figure 3. pdf graph for $n=10$

From Figure. 1, $2 \& 3$ different shapes of the pdf of lower record values from size-biased power function distribution can be observed.

1. In Figure 1 for $\alpha=7, n=2, \beta=0.5 \& 1$ the shape of the LR-SPFD is flatter and showing longer right tail but for $\alpha=7, n=2, \beta=5,7 \& 10$ showing longer left tail and peaked.

2. In Figure $2 \alpha=7, n=5, \beta=0.5 \& 1$ the pdf of LR-SPFD is showing longer right tail (positively skewed). For $\alpha=7, n=5, \beta=5,7 \& 10$ the pdf is approximately symmetric.

3. In Figure $3 \alpha=10, n=10, \beta=5 \& 7$ the pdf of LR-SPFD is showing longer right tail (positively skewed) but for $\alpha=10, n=10, \beta=0.5 \& 1$ highly positively skewed and flattered. For $\alpha=10, n=10, \beta=10$, the pdf is approximately symmetric.

The cumulative distribution function LR-SPFD

$$
F(x)=\frac{\Gamma\left(n, x^{\prime}\right)}{\Gamma(n)}
$$

Where $\Gamma\left(n, x^{\prime}\right)=\int_{x^{\prime}}^{\infty} t^{n-1} e^{-t} d t$ is incomplete gamma function and $x^{\prime}=-(\beta+1) \ln (x / \alpha)$

\subsection{Moments}

The $r^{\text {th }}$ moments of the LR-SPFD are

$$
\mu_{r(n)}^{\prime}=\alpha^{r}\left(\frac{\beta+1}{\beta+r+1}\right)^{n}
$$

First four raw moments of the LR-SPFD

$$
\mu_{1(n)}^{\prime}=\alpha\left(\frac{\beta+1}{\beta+2}\right)^{n}, \mu_{2(n)}^{\prime}=\alpha^{2}\left(\frac{\beta+1}{\beta+3}\right)^{n}, \mu_{3(n)}^{\prime}=\alpha^{3}\left(\frac{\beta+1}{\beta+4}\right)^{n}, \mu_{4(n)}^{\prime}=\alpha^{4}\left(\frac{\beta+1}{\beta+5}\right)^{n}
$$

Mean, variance, $3^{\text {rd }}$ and $4^{\text {th }}$ mean moments of the LR-SPFD

$$
\begin{aligned}
& E\left(X_{L(n)}\right)=\mu_{1(n)}^{\prime}=\alpha\left(\frac{\beta+1}{\beta+2}\right) \\
& \operatorname{Var}\left(X_{L(n)}\right)=\mu_{2(n)}=\alpha^{2}\left[\left(\frac{\beta+1}{\beta+3}\right)^{n}-\left(\frac{\beta+1}{\beta+2}\right)^{2 n}\right] \\
& \mu_{3(n)}=\alpha^{3}(\beta+1)^{n}\left[\left(\frac{1}{\beta+4}\right)^{n}-3 \frac{(\beta+1)^{n}}{(\beta+2)^{n}(\beta+3)^{n}}+2 \frac{(\beta+1)^{2 n}}{(\beta+2)^{3 n}}\right] \\
& \mu_{4(n)}=\alpha^{4}(\beta+1)^{n}\left[\frac{1}{(\beta+5)^{n}}-\frac{4(\beta+1)^{n}}{(\beta+2)^{n}(\beta+4)^{n}}+\frac{6(\beta+1)^{2 n}}{(\beta+2)^{2 n}(\beta+3)^{n}}-\frac{3(\beta+1)^{3 n}}{(\beta+2)^{4 n}}\right]
\end{aligned}
$$

Coefficient of skewness and kurtosis of the LR-SPFD 


$$
\begin{gathered}
\beta_{1}=\frac{\left[(\beta+2)^{3 n}(\beta+1)-3(\beta+2)^{2 n}(\beta+4)^{n}+2(\beta+1)^{2 n}(\beta+3)^{n}(\beta+4)^{n}\right]}{(\beta+1)^{n}(\beta+2)^{n}(\beta+4)^{n}\left[(\beta+2)^{2 n}-(\beta+1)^{n}(\beta+3)^{n}\right]} \\
\beta_{2}=\frac{(\beta+2)^{2 n}(\beta+3)^{n}(\beta+4)^{n}-4(\beta+1)^{n}(\beta+2)^{3 n}(\beta+3)^{n}(\beta+5)^{n}}{(\beta+1)(\beta+2)^{2 n}(\beta+4)^{n}(\beta+5)^{n}\left[(\beta+2)^{2 n}-(\beta+1)^{n}(\beta+3)^{n}\right]} \\
+\frac{6(\beta+1)^{2 n}(\beta+2)^{2 n}(\beta+4)^{n}(\beta+5)^{n}-3(\beta+1)^{3 n}(\beta+3)^{n}(\beta+4)^{n}(\beta+5)^{n}}{(\beta+1)(\beta+2)^{2 n}(\beta+4)^{n}(\beta+5)^{n}\left[(\beta+2)^{2 n}-(\beta+1)^{n}(\beta+3)^{n}\right]}
\end{gathered}
$$

From equations (17) and (18) it can be seen that the coefficient of skewness and kurtosis are independent from scale parameter $\alpha$

Table 1. Values of coefficient of skewness for $n=5 \&$ different values of shape parameter $\beta$

\begin{tabular}{|c|c|}
\hline \multicolumn{2}{|c|}{$\mathbf{n = 5}$} \\
\hline $\begin{array}{c}\beta \\
\text { Shape Parameter }\end{array}$ & $\begin{array}{c}\beta_{1} \\
\text { Skewness }\end{array}$ \\
\hline 2 & 0.00509 \\
\hline 5 & 0.00109 \\
\hline 7 & 0.00053 \\
\hline 10 & 0.00023 \\
\hline 13 & 0.00012 \\
\hline 15 & 0.00008 \\
\hline 20 & 0.00004 \\
\hline 50 & 0.00000 \\
\hline
\end{tabular}

From Table 1, it can be seen that as shape parameter $\beta$ increases the coefficient of skewness is decreases and approaches to zero. From table 1 and Figure 1,2\&3 it can be concluded that the shape of the LR-SPFD is approaching to symmetric for $\beta \geq 5, n \geq 5$.

\subsection{Entropy}

The Shannon entropy of the LR-SPFD is

$$
H(x)=(\beta+1) \ln (\alpha)+\ln (\Gamma n)+\frac{n \beta}{(\beta+1)}-\beta \ln (\alpha)-\ln (\beta+1)-(n-1) \Psi^{(0)}(n)
$$

where $\int_{0}^{\infty} t^{n-1} \ln (t) e^{-t} d t=\Gamma(n) \Psi^{0}(n)$, for $\operatorname{Re}(n)>0$, and $\Psi^{(0)}(n)=\Psi(n)$ is the $n^{t h}$ derivative of the digamma function.

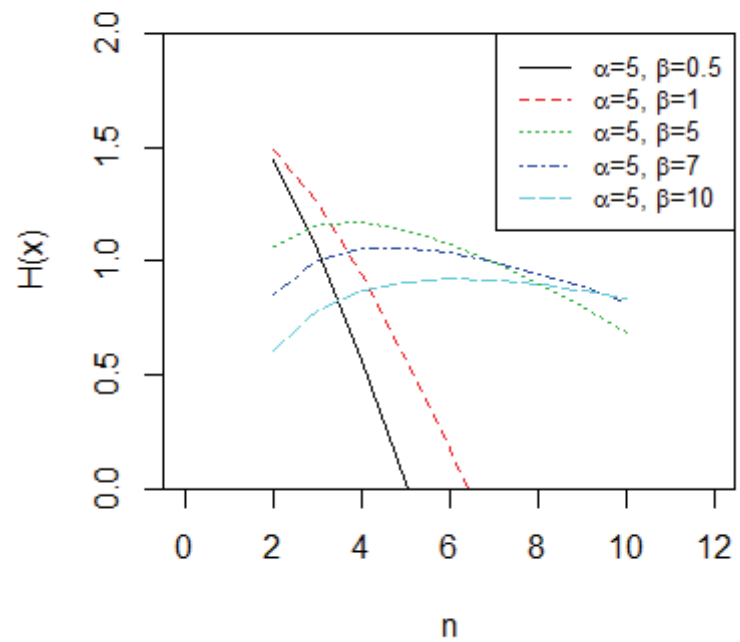

Figure 4. entropy graph for $n=2,3,4,5,6,7,8,9,10$

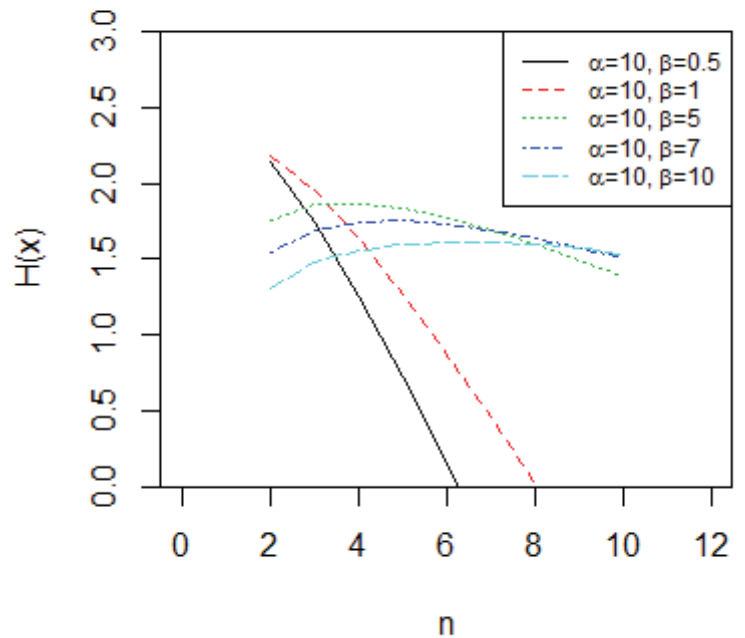

Figure 5. entropy graph for $n=2,3,4,5,6,7,8,9,10$ 


\subsection{Reliability Measures}

The survival function of the LR-SPFD

$$
S_{n}(x)=\frac{\gamma\left(n, x^{\prime}\right)}{\Gamma(n)}
$$

Where $\gamma\left(n, x^{\prime}\right)=\int_{0}^{x^{\prime}} t^{n-1} e^{-t} d t$ is incomplete gamma function and $x^{\prime}=-(\beta+1) \ln (x / \alpha)$

The hazard rate function of the LR-SPFD

$$
h_{n}(x)=\frac{(\beta+1)^{n} x^{\beta}[-\ln (x / \alpha)]^{n-1}}{\alpha^{\beta+1} \gamma\left(n, x^{\prime}\right)}
$$

\section{Joint Record Values from the Size-Biased Power Function Distribution}

Using equations (7) and (8) in equation (2), we get the joint pdf of the lower record values from SPFD

$$
f_{n, m}(x, y)=\frac{(\beta+1)^{n} y^{\beta}}{\alpha^{\beta+1}(m-1) !(n-m-1) ! x}[-\ln (x / \alpha)]^{m-1}[\ln (x / \alpha)-\ln (y / \alpha)]^{n-m-1}, \quad 0<y<x<\alpha, m<n, y<x
$$

\section{Recurrence Relations for Moments}

In this section some recurrence relations satisfied by the single and product moments of the lower record values from the SPFD are given. It is found that the size-biased power function distribution (SPFD) having the following relationship between pdf and cdf,

$$
f(x)=\frac{(\beta+1) F(x)}{x}
$$

Theorem 1: for $n>1$ and $r=0,1,2,3, \ldots$

$$
E\left(X_{L(n-1)}\right)^{r}=E\left(X_{L(n)}\right)^{r}\left(\frac{\beta+r+1}{\beta+1}\right)
$$

Proof: Let $X_{L(n)}$ from LR-SPFD in equation (9) and , for $n>1$ and $r=0,1,2,3, \ldots$

$E\left(X_{L(n)}^{r}\right)=\int_{0}^{\alpha} x^{r} f_{n}(x) d x$

$$
E\left(X_{L(n)}^{r}\right)=\int_{0}^{\alpha} x^{r} \frac{[-\ln F(x)]^{n-1}}{(n-1) !} f(x) d x
$$

after some simplifications we get

$$
E\left(X_{L(n)}^{r}\right)=\frac{(\beta+1)(n-1)}{r(n-2) !} \int_{0}^{\alpha} x^{r}[-\ln F(x)]^{n-1} f(x) d x-\frac{(\beta+1)}{r(n-1) !} \int_{0}^{\alpha} x^{r}[-\ln F(x)]^{n-1} f(x) d x
$$

The relation in equation (24) is developed by simply rewriting equation (25).

Theorem 2 For $i<m<n-2$ and $r, s=0,1,2,3, \ldots$

$$
E\left[X_{L(m)}^{r} X_{L(n-1)}^{s}\right]=\left(\frac{\beta+s+1}{\beta+1}\right) E\left[X_{L(m)}^{r} X_{L(n)}^{s}\right]
$$

and for $i<m<n-1$ and $r, s=0,1,2,3, \ldots$

$$
E\left(X_{L(m)}^{r} X_{L(m+1)}^{s}\right)=\frac{\beta+1}{\beta+s+1} E\left(X_{L(m)}^{r+s}\right)
$$

Proof: Let $X_{L(n)}$ and $X_{L(n)}$ from LR-SPFD in equation (22) and, for $i<m<n-2$ and $r, s=0,1,2,3, \ldots$

$$
E\left(X_{L(m)}^{r} X_{L(n)}^{s}\right)=E\left(x^{r} y^{s}\right)=\frac{1}{(m-1) !(n-m-1) !} \int_{0}^{\alpha} x^{\alpha}[-\ln F(x)]^{m-1} \frac{f(x)}{F(x)} l(x) d x
$$




$$
\begin{aligned}
& l(x)=\int_{0}^{\alpha} y^{s}[-\ln F(y)+\ln F(x)]^{n-m-1} f(y) d y \\
& l(x)=(\beta+1) \int_{0}^{\alpha} y^{s-1}[-\ln F(y)+\ln F(x)]^{n-m-1} F(y) d y \\
& l(x)=\frac{(\beta+1)(n-m-1)}{s} \int_{0}^{\alpha} y^{s}[-\ln F(y)+\ln F(x)]^{n-m-2} f(y) d y-\frac{(\beta+1)}{s} \int_{0}^{\alpha} y^{s}[-\ln F(y)+\ln F(x)]^{n-m-1} f(y) d y
\end{aligned}
$$

Substituting equation (29) in (28) and simplifying and rewriting it, we get equation (26) for $i<m<n-2$ and equation (27) for $i<m<n-1$.

Characterization: In this section a characterization of the LR-SPFD is presented.

Theorem 3: Let $X_{1}, X_{2}, \ldots, X_{n}(n \geq 1)$ be independent and identically distributed random variables having continuous cdf $F(x)$, where $0 \leq F(x) \leq 1$, for all $0<x<\alpha$. Assuming that $\mathrm{F}(\mathrm{x})$ is twice differentiable,

$$
E\left[\left\{X_{L(m+1)}-X_{L(m)}\right\}^{2} / X_{L(m)}\right]=\frac{2 x^{2}}{(\beta+2)(\beta+3)}
$$

where $X \in S P F D(0, \alpha)$.

Proof: using the conditional pdf of lower record values in eq. (4), where $\mathrm{F}(\mathrm{x})$ and $\mathrm{F}(\mathrm{x})$ are the cdf and pdf from SPFD, we have

$$
\int_{0}^{x}(y-x)^{2} f(y) d y=\frac{2 x^{2}}{(\beta+2)(\beta+3)} F(x)
$$

Differentiating both sides of equation (31) we get

$$
\int_{0}^{x}-2(y-x) f(y) d y=\frac{4 x F(x)}{(\beta+2)(\beta+3)}+\frac{2 x^{2} f(x)}{(\beta+2)(\beta+3)}
$$

Again differentiating equation (32) we get

$$
2 F(x)=\frac{4 F(x)}{(\beta+2)(\beta+3)}+\frac{8 x f(x)}{(\beta+2)(\beta+3)}+\frac{2 x^{2} f^{\prime}(x)}{(\beta+2)(\beta+3)}
$$

Substituting $F(x)=y, f(x)=y^{\prime}, f^{\prime}(x)=y^{\prime \prime}$ in equation (33)

$$
x^{2} y^{\prime \prime}+4 x y^{\prime}-\left(\beta^{2}+5 \beta+4\right) y=0
$$

Equation (34) is renowned Euler-type equation. This equation has the solution of the form $y=x^{r}$, where r must satisfy the equation

$$
r^{2}+3 r-\left(\beta^{2}+5 \beta+4\right)=0
$$

For the above equation the roots of $\mathrm{r}$ are $-(\beta+4) \&(\beta+1)$. So the solutions are of the type $y=c_{1} x^{-(\beta+4)} \& y=c_{2} x^{\beta+1}$, where $c_{1}$ and $c_{2}$ are constants. We have

$$
F(0)=0 \quad \text { and } \quad F(\alpha)=1
$$

Since the solution $y=c_{1} x^{-(\beta+4)}$ does not satisfy the condition eq. (36) and $y=c_{2} x^{\beta+1}=F(x)$ where $c_{2}=\frac{1}{\alpha^{(\beta+1)}}$, satisfies the condition in eq. (36), so we must have,

$$
y=F(x)=x^{\beta+1}
$$

Characterization is a classic property of probability models. Probability distributions can be characterized by many methods among them one is cdf technique. Many researchers characterized probability distributions using conditional pdf of lower/upper record values by cdf technique mentioned in the introduction section. It can be observed that the lower record values from size-biased power function distribution showing the such elegant properties as other well know probability distributions having. The reliability measures as survival function and hazard function derived from record value theory have many real life applications. Moreover, the recurrence relations from the LR-SPFD have been developed. These relations can be used to obtain all single and product moments of record values form the SPFD in simple recursive manner. 


\section{Conclusion}

Record value theory has been derived on various well-known continuous distributions but no work has been done on record values from weighted or size-biased distributions till the paper Bashir and Ahmad (2014) record values from the size biased Pareto distribution. In this paper lower record values have been developed from size-biased power function distribution. Various properties of the LR-SPFD have been derived including graphical trend of the pdf of LR-SPFD. It is concluded that as shape parameter increases the pdf approaches to symmetry. Shannon entropy and reliability measures of the LR-SPFD have also been derived. Some recurrence relations of single and product moments of LR-SPFD have been developed that can be used to obtain all single and product moments of the LR-SPFD. A characterization of the LR-SPFD is derived by using cumulative distribution function.

\section{References}

Ahsanullah, M., \& Bhoj, D. S. (1996). Record Values of Extreme Value Distributions and a Test for Domain of Attraction of Type-I Extreme Value Distribution. Sankhya, 58, 151-158

Ahsanullah, M., \& Houchens, R. L. (1989). A Note on Record Values from a Pareto Distribution. Pak. J. Statist, $5,51-57$.

Ahsanullah, M., \& Lutful Kabir, A. B. M. (1974). A characterization of the power function distribution. The Canadian Journal of statistics, 2(1), 95-98.

Ahsanullah, M. (2010). Concomitants of Upper Record Statistics for Bivariate Pseudo- Weibull Distribution. Applications and Applied Mathematics, 5(10), 1379- 1388.

Ahsanullah, M. (1984). Linear Prediction of Record Values for the Two Parameter Exponential Distribution. Ann. Inst. Statist. Math., 32, 363-368.

Ahsanullah, M. (1992). Record Values of Independent and Identically Distributed Continuous Random Variables. Pak. J. Statist., 8(2), 9-34.

Ahsanullah, M., Shakil, M., \& Kibria, B. M. G. (2013). A characterization of power function distribution based on lower record values. Prob. Stat Forum, 6, 68-72.

Ahsanullah, M. (1986). Record Values from a Rectangular Distribution. Pak. J. Statist., 2(1), 1-5.

Balakrishnan, N., \& Chan, P. S. (1998). On the Normal Record Values and Associated Inference. Elsevier, 39, 73-80.

Balakrishnan, N., \& Chan, P. S. (1993). Record Values from Rayleigh and Weibull Distribution and Associated Inference. Nat. Inst. Stand. Technol. J. Res. Spec. Publ., 866, 41-51.

Balakrishnan, N., Ahsanullah, M., \& Chan, P. S. (1995). On the Logistic Record Values and Associated Inference. J. App. Statist. Sci., 2, 233-248.

Bashir, S., \& Ahmad, M. (2014). On the inverse Gaussian record values. International journal of engineering sciences and research technology, 3(7), 729-737.

Bashir, S., \& Ahmad, M. (2014). Record values from the size-biased Pareto distribution and a characterization. International Journal of Engineering Research and General Science, 2(4), 101-109.

Bashir, S., \& Ahmad, M. (2014). Record values from the size-biased Pareto distribution and a characterization. International Journal of Engineering Research and General Science, 2(4), 101-109.

Bashir, S., \& Ahmad, M. (2015). Recurrence relations for single and product moments of record values from size-biased Pareto distribution. International journal of economic and business review, 3(5), 139-144.

Bashir, S., \& Akhtar, K. (2014). Record values on the size-biased students t distribution. International Journal of Innovative Science, Engineering $\mathcal{E}$ Technology, 1(5), 344-348.

Bashir, S., \& Rasul, M. (2015). A note on lower record values from the weighted rectangular distribution. International Journal of Advanced Scientific and Technical Research, 3(5), 413-419.

Bashir, S., Ahmed, K., \& Ahmad, M. (2014). A Note on Record Values from a Two-Sided Power Distribution. Pak Journal of Statistics, 30(2), 245-252.

Chandler, K. N. (1952). The Distribution and Frequency of Record Values. J. Roy. Statist. Soc., 14, $220-228$.

Khan, R. U., \& Zia, B. (2009). Recurrence Relations for Single and Product Moments of Record values from Gompertz Distribution and a Characterization. World Applied Sciences Journal, 7(10), 1331-1334.

Lim, E., \& Lee, M. (2013). A characterization of the power function distribution by independent property of lower record 
values. Journal of the chungcheong mathematical society, 26(2),269-273.

Patil, G. P., \& Rao, C. R. (1977). Weighted distributions: a survey of their application. In P. R. Krishnaiah (Ed.), Applications of Statistics, 383-405. North Holland Publishing Company.

Raqab, M. Z. (2000). On the Moments of Record Values. Commun. In Statist. Theo. And Meth, 29(7), 1631-1647.

Saran, J., \& Pandey, A. (2004). Estimations of parameters of a power function distribution and its characterization by kth record values. STATISTICA, anno LXIV, n.3, 523-536.

Shakil, M., \& Ahsanullah, M. (2011). Record Values of the Ratio of Rayleigh Random Variables. Pak. J. Statist., 27(3), 307-325.

Sultan, K. S. (2010). Record Values from the Inverse Weibull Lifetime Model: Different Methods of Estimation. Intelligence Information Management, 2, 631-636.

Sultan, K. S., Al-Dayian, G. R., \& Mohammad, H. H. (2008). Estimation and Prediction from Gamma Distribution based on Record Values. Computational Statistics and Data Analysis, 52, 1430-1440.

Teimouri, M., \& Gupta, A. K. (2012). On the Weibull Record Statistics and Associated Inference. Statistica, $2,145-162$.

\section{Copyrights}

Copyright for this article is retained by the author(s), with first publication rights granted to the journal.

This is an open-access article distributed under the terms and conditions of the Creative Commons Attribution license (http://creativecommons.org/licenses/by/4.0/). 